\title{
SPIROGYRA LINK AND SIROGONIUM KÜTZING SPECIES: NEW TO ALGAL FLORA OF NEPAL
}

\author{
S.K. Rai and P.K. Misra* \\ Department of Botany \\ Post Graduate Campus, Tribhuvan University, Biratnagar, Nepal \\ Email: shivarai2003@yahoo.com \\ *Phycology Research Laboratory \\ Department of Botany, Lucknow University, Lucknow-226007, India
}

\begin{abstract}
Eleven species of Spirogyra Link and two species of Sirogonium Kützing have been described in this communication as new records for Nepal. These are Spirogyra communis (Hassal) Kützing, $S$. daedaleoides Czurda, S. decimina (Müller) Kützing, S. esthonica (Skuja) Czurda, S. juergensii Kützing, S. paradoxa Rao, S. pratensis Transeau, S. rhizopus Jao, S. rugulosa Iwanoff, S. spreeiana Rabenhorst's, S. verrucosa (Rao) Krieger, Sirogonium illinoiense (Transeau) G.M. Smith and $S$. sticticum (Engl. Bot.) Kützing. All these taxa were collected from the lotic and lentic habitats of eastern Nepal. The genus, Sirogonium, is being described for the first time from this country.
\end{abstract}

Key words: Algae, chlorophyceae, zygnemataceae, Spirogyra, Sirogonium.

\section{INTRODUCTION}

Our knowledge of zygnemataceae specially of the genera Spirogyra Link and Sirogonium Kützing in Nepal is inadequate. So far, only one species of Spirogyra i.e., S. nepalensis nov. sp. is found to be reported from Lobuche by Müller (1965). The information regarding Sirogonium and its distribution in Nepal is completely lacking. Therefore, it was felt desirable to study the zygnematacean flora of Nepal.

The present paper describes the morphology and distribution of Spirogyra and Sirogonium species belonging to the family zygnemataceae under the class chlorophyceae, which are unreported from Nepal hitherto. All these taxa were observed by the authors in the course of studies on freshwater algal diversity of eastern Nepal.

\section{MATERIAL AND METHODS}

Between 2002-2004, samples were collected using a plankton-net of mesh size $80 \mu \mathrm{m}$ and preserved in $4 \%$ unbuffered formalin. Detailed morpho-taxonomic study and photomicrography were done under Nikon Labophot microscope with camera attachment. Identification of species was by consulting various literatures and monographs and the classification followed Smith (1933). Species under each genus are enumerated alphabetically.

\section{SYSTEMATIC DESCRIPTIONS}

Class: Chlorophyceae

Order: Zygnematales

Family: Zygnemataceae

Genus: Spirogyra Link 1820 
Filaments free floating, rarely attached, simple, long, unbranched, covered with mucilaginous sheath, without basal-distal differentiation; vegetative cells cylindrical, $1 / 2-30$ times as long as broad; cross walls plane, colligate, semi-replicate, replicate or unduliseptate; chloroplasts 1-16, spirally arranged, parietal band or ribbon shaped, $1 / 2-3$ (rarely more than 8 ) turns, often straight, each with numerous prominent pyrenoids; nucleus centrally placed, supported by several cytoplasmic strands.

Reproduction by zygospores, aplanospores, parthenospores, akinetes and fragmentation; conjugation usually scalariform, sometimes laterai; zygospores usually ellipsoid, rarely ovoid or lenticular; median spore wall chitinous, smooth or variously ornamented, pale yellow to chestnut brown.

Spirogyra is more or less universal in distribution and more than 289 species have been described in the world (Randhawa, 1959).

\section{Key to the species:}

1. Cross wall plane 2

1. Cross wall replicate, gametangia fusiform, zygospore ellipsoid, vegetative cells $18-24 \mu \mathrm{m}$ broad ------ S. spreeiana (10)

2. Conjugation tube formed wholly or largely by male gametangia, chloroplast 1

$-3$

2. Conjugation tube formed by both gametangia, chloroplast 1 to many $-4$

3. Zygospore laterally compressed, median spore wall irregularly reticulate, vegetative cells 26 $39 \mu \mathrm{m}$ broad ---

3. Zygospore ellipsoid, median spore wall punctate, vegetative cells $48-52 \mu \mathrm{m}$ broad ------

$$
\text { S. rugulosa (9) }
$$

4. Chloroplast 1

4. Chloroplast many $-8$
5. Median spore wall smooth $-6$

5. Median spore wall irregularly reticulate, zygospore ellipsoid or pear shaped, vegetative cells $34-45 \mu \mathrm{m}$ broad ------ $S$. daedaleoides (2)

6. Gametangia cylindrical -...-_..-_

6. Gametangia swollen, vegetative cells $18-20 \mu \mathrm{m}$ broad --.- S. pratensis (7)

7. Vegetative cells $17-20 \mu \mathrm{m}$ broad S. communis (1)

7. Vegetative cells $24-34 \mu \mathrm{m}$ broad

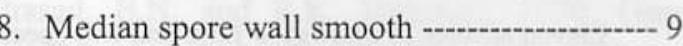

8. Median spore wall variously ornamented ---10

9. Gametangia nearly cylindric, zygospore ovoid to globose, chloroplasts $2-3$, vegetative cells 37-42 $\mu \mathrm{m}$ broad ------------ S. decimina (3)

9. Gametangia swollen, zygospore broadly ellipsoid to almost spherical, chloroplasts $3-4$, vegetative cells $80-90 \mu \mathrm{m}$ broad

S. paradoxa (6)

10. Median spore wall irregularly reticulate, zygospore ovoid, chloroplasts 2 , vegetative cells $40 \mu \mathrm{m}$ broad S. rhizopus (8)

10. Median spore wall verrucose, zygospore ellipsoid, chloroplasts 4-8, vegetative cells 110 $130 \mu \mathrm{m}$ broad -...-...- S. verrucosa (11)

Spirogyra communis (Hassal) Kützing 1849 (Pl. 1, Fig. 8)

Prescott, G.W. 1951, P. 312; Tiffany, L.H. and M.E. Britton 1952, P. 146, Pl. 43, Fig. 455; Randhawa, M.S. 1959, P. 293, Fig. 247

Vegetative cells $40-69 \mu \mathrm{m}$ long, $17-20 \mu \mathrm{m}$ broad, with plane end walls; chloroplast $1,1 / 2-4$ turns; conjugation scalariform, tubes formed by both gametangia; empty cells 40-60 $\mu \mathrm{m}$ long, $17 \mu \mathrm{m}$ broad; fruiting cells cylindrical, $62-65 \mu \mathrm{m}$ long, $17.5 \mu \mathrm{m}$ broad; zygospores ellipsoid, $37-40 \mu \mathrm{m}$ long, $17-19 \mu \mathrm{m}$ broad; median spore wall smooth, yellow. 
Locality: Free floating in a reservoir near Koshi Barrage, $162 \mathrm{~m}$, Bhantabari, Sunsari district.

Collection number and date: EN 128, 29-032003.

Distribution: India (Gorakhpur, Assam), U.S.A. (New Caledonia).

Spirogyra daedaleoides Czurda 1932 (P1. 1, Fig. 5)

Prescott, G.W. 1951, P. 313, Pl. 72, Figs. 9-11; Tiffany, L.H. and M.E. Britton 1952, P. 148, P1. 45, Figs. 473-474; Randhawa, M.S. 1959, P. 377 , Fig. 416

Vegetative cells $60-85 \mu \mathrm{m}$ long, $34-45 \mu \mathrm{m}$ broad, with plane end walls; chloroplast 1, 2-8 turns; conjugation scalariform and lateral; empty cells 60-65 $\mu \mathrm{m}$ long, $47 \mu \mathrm{m}$ broad; fruiting cells slightly inflated, $70 \mu \mathrm{m}$ long, $55 \mu \mathrm{m}$ broad; zygospores ellipsoid or pear shaped, $65-70 \mu \mathrm{m}$ long, $42-49 \mu \mathrm{m}$ broad; median spore wall with irregularly reticulate ridges, brown.

Locality: Free floating in the roadside pool at Itahari, $120 \mathrm{~m}$, Sunsari district.

Collection number and date: EN 303, 10-092004.

Distribution: Latvia, U.S.A.

Spirogyra decimina (Müller) Kützing 1843 (Pl. 1, Fig. 9)

Prescott, G.W. 1951, P. 313; Tiffany, L.H. and M.E. Britton 1952, P. 153, Pl. 46, Fig. 489; Randhawa, M.S. 1959, P. 325, Figs. 309a-b; Prasad, B.N. and P.K. Misra 1992, P. 80, Pl. 13, Figs. 4-5

Vegetative cells $55.5-105 \mu \mathrm{m}$ long, 37-42 $\mu \mathrm{m}$ broad, with plane end walls; chloroplasts 2-3, 1-2 turns; conjugation scalariform, tubes formed by both gametangia; empty cells $55-70 \mu \mathrm{m}$ long, 37.5 $\mu \mathrm{m}$ broad; fruiting cells slightly inflated, 98-102 $\mu \mathrm{m}$ long, 48-53 $\mu \mathrm{m}$ broad; zygospores ovoid to globose, 58-60 $\mu \mathrm{m}$ long, 35-38 $\mu \mathrm{m}$ broad; median spore wall smooth, yellow.

Locality: Floating on the edge of Muga river on the way from Hile to Pakhribas, $1850 \mathrm{~m}$, Dhankuta district.

Collection number and date: EN 78, 20-10-2002.

Distribution: Africa, Asia (India: Banaras, Bareilly, Gorakhpur, Hosangabad, Kerala, Port
Blair, Srinagar; Java; Myanmar: S. decimina var. plena West and West from Kyauktaga of Pegu district and Singaing of Kyaukse district), Europe, South America, U.S.A., West Indies.

Spirogyra esthonica (Skuja) Czurda 1932 (PI. 1, Figs. 3-4)

Randhawa, M.S. 1959, P. 396, Fig. 460

Vegetative cells 140-168 $\mu \mathrm{m}$ long, 26-39 $\mu \mathrm{m}$ broad, with plane end walls; chloroplast $1,7.5-8$ turns; conjugation scalariform, tubes formed largely by male gametangia, sterile cells present between gametangia; empty cells 138-140 $\mu \mathrm{m}$ long, 27-30 $\mu \mathrm{m}$ broad; fruiting cells inflated, 186$190 \mu \mathrm{m}$ long, 43-50 $\mu \mathrm{m}$ broad; zygospores ellipsoid, laterally compresses, $80-85 \mu \mathrm{m}$ long, 42$49 \mu \mathrm{m}$ broad; median spore wall irregularly corrugate with minute punctations between the ridges.

Locality: Free-floating in Mechi pond, $80 \mathrm{~m}$, Maheshpur, Bhadrapur, Jhapa district.

Collection number and date: EN 269, 17-062004.

Distribution: Estonia.

Spirogyra juergensii Kützing 1845 (Pl. 1, Fig. 10)

Prescott, G.W. 1951, P. 316, Pl. 73, Figs. 7-8; Tiffany, L.H. and M.E. Britton 1952, P. 146, Pl. 44, Fig. 463; Randhawa, M.S. 1959, P. 294, Fig. 250

Vegetative cells $90-115 \mu \mathrm{m}$ long, 24-34 $\mu \mathrm{m}$ broad, with plane and occasionally swollen end walls; chloroplast 1, 2-4 turns; conjugation scalariform, tubes formed by both gametangia; empty cells 92 $105 \mu \mathrm{m}$ long, $28-29 \mu \mathrm{m}$ broad; fruiting cells cylindrical, 90-95 $\mu \mathrm{m}$ long, 35-36 $\mu \mathrm{m}$ broad; zygospores ellipsoid, $60-68 \mu \mathrm{m}$ long, 30-32 $\mu \mathrm{m}$ broad; median spore wall smooth, yellow.

Locality: Free-floating in a reservoir near Koshi Barrage, $162 \mathrm{~m}$, Bhantabari, Sunsari district.

Collection number and date: EN 128, 29-032003.

Distribution: Australia, Europe, India (Gorakhpur), Pakistan (Lahore), South America, U.S.A.

Spirogyra paradoxa Rao 1937 (Pl. 2, Fig. 3)

Randhawa, M.S. 1959, P. 326, Fig. 313; Rattan, R.S. 1967, P. 98, Figs. 15-16; Prasad, B.N. and P.K. Misra 1992, P. 85, Pl. 13, Figs. 8 and 10. 
Vegetative cells short, $50-70 \mu \mathrm{m}$ long, $80-90 \mu \mathrm{m}$ broad, with plane end walls; chloroplasts $3-4$; conjugation scalariform; empty cells $50-56 \mu \mathrm{m}$ long, $82-90 \mu \mathrm{m}$ broad; fruiting cells slightly inflated, $80-90 \mu \mathrm{m}$ long, $96 \mu \mathrm{m}$ broad; zygospore broadly ellipsoidal to almost spherical, $80-85 \mu \mathrm{m}$ long, 68-72 $\mu \mathrm{m}$ broad; median spore wall smooth, brown.

Locality: Floating with other algae in Pitchhra pond, $72 \mathrm{~m}$, Biratnagar, Morang district.

Collection number and date: EN 84, 16-12-2002.

Distribution: India (Dehradun, Meerut, Port Blair, Punjab, Sarnath).

Spirogyra pratensis Transeau 1914 (Pl. 1, Fig. 11)

Prescott, G.W. 1951, P. 319, Pl. 75, Figs. 4-6; Tiffany, L.H. and M.E. Britton 1952, P. 144, Pl. 43, Fig. 457; Randhawa, M.S. 1959, P. 297, Fig. 257; Prasad, B.N. and P.K. Misra 1992, P. 87, P1. 14, Figs. 5 and 7.

Vegetative cells 100-135 $\mu \mathrm{m}$ long, 18-20 $\mu \mathrm{m}$ broad, with plane end walls; chloroplast 1 (rarely 2), 1-8 turns; conjugation scalariform and lateral, tubes formed by both gametangia; empty cells 105-120 $\mu \mathrm{m}$ long, $20 \mu \mathrm{m}$ broad; fruiting cells fusiform-inflated, $95 \mu \mathrm{m}$ long, 38-42 $\mu \mathrm{m}$ broad; sterile cells cylindrical; zygospores usually ellipsoid, sometimes ovoid or cylindric ovoid, 56 $\mu \mathrm{m}$ long, $29 \mu \mathrm{m}$ broad; median spore wall smooth, yellow.

Remarks: Bullate-shaped inflatations of sterile cells are not observed in the present specimen but in other respects resemble to the type.

Locality: Free-floating in Raja Rani lake, 700 m, Bhogateni, Morang district.

Collection number and date: EN 113, 11-012003.

Distribution: China (Nanking, Peiping), India (Banaras, Port Blair, Punjab), U.S.A.

Spirogyra rhizopus Jao 1936 (P1. 1, Figs. 6-7)

Randhawa, M.S. 1959, P. 331, Figs. 321a-c

Vegetative cells $160-175 \mu \mathrm{m}$ long, $40 \mu \mathrm{m}$ broad, with plane end walls; basal cell expanded with irregularly lobed holdfast; chloroplasts $2,1 / 2-4$ turns; conjugation scalariform, tubes formed by both gametangia; empty cells $70-80 \mu \mathrm{m}$ long, 40 $\mu \mathrm{m}$ broad; fruiting cells quadrangularly inflated,
90-95 $\mu \mathrm{m}$ long, 40-50 $\mu \mathrm{m}$ broad; zygospores ellipsoid, $58-60 \mu \mathrm{m}$ long, $38-40 \mu \mathrm{m}$ broad; outer spore wall thick, lamellose, hyaline; median spore wall irregularly reticulate, brown.

Locality: Attached on moist stones around the tap, $520 \mathrm{~m}$, Bhandare Ghat, Sun Koshi, Udayapur district.

Collection number and date: EN 191, 24-052003.

Distribution: China (Peiping).

Spirogyra rugulosa Iwanoff 1902 (P1. 1, Figs. 1-2)

Tiffany, L.H. and M.E. Britton 1952, P. 156, Pl. 47, Fig. 498; Randhawa, M.S. 1959, P. 352, Fig. 365.

Vegetative cells $90-160 \mu \mathrm{m}$ long, $48-52 \mu \mathrm{m}$ broad, with plane end walls; chloroplast 1, 5-6 turns; conjugation scalariform, tubes formed by male gametangia; empty cells 80-95 $\mu \mathrm{m}$ long, $52-55 \mu \mathrm{m}$ broad; fruiting cells both short and long, inflated on conjugating side, 50-118 $\mu \mathrm{m}$ long, 55-70 $\mu \mathrm{m}$ broad; zygospore ellipsoid, $70 \mu \mathrm{m}$ long, 38-45 $\mu \mathrm{m}$ broad; median spore wall finely punctate, yellowbrown.

Remarks: In the present algae, zygospores are small in size mainly on length and their shapes are not ovoid with broadly rounded ends as described in type specimen. Also, not all zygospore bearing cells are shortened otherwise in other respects resemble the type.

Locality: Planktonic in the roadside pool near P.G. Campus, 72 m, Biratnagar, Morang district.

Collection number and date: EN 224, 24-072003.

Distribution: China, Rumania, Russia, U.S.A.

Spirogyra spreeiana Rabenhorst's 1863 (Pl. 2, Fig. 2)

Prescott, G.W. 1951, P. 321, Pl. 77, Fig. 9; Tiffany, L.H. and M.E. Britton 1952, P. 158, Pl. 48, Fig. 513; Randhawa, M.S. 1959, P. 355, Fig. 370

Vegetative cells 100-142 $\mu \mathrm{m}$ long, 18-24 $\mu \mathrm{m}$ broad, with replicate end walls; chloroplast $1,1 / 2-4$ turns; conjugation scalariform and lateral; empty cells $19 \mu \mathrm{m}$ broad; fruiting cells inflated, $112 \mu \mathrm{m}$ long, $35 \mu \mathrm{m}$ broad; zygospores ellipsoid, $75 \mu \mathrm{m}$ long, 30-32 $\mu \mathrm{m}$ broad; median spore wall smooth, yellow.

ECOPRINT VOL 14, 2007 
Remarks: The vegetative cells of this algae are shorter than the type.

Locality: Free-floating in Phooli Khola stream near Damku Village, 1690 m, Khotang district.

Collection number and date: EN 175, 18-052003.

Distribution: Finland, Germany, India, Rumania, South Africa, U.S.A.

Spirogyra verrucosa (Rao) Krieger 1944 (Pl. 2, Fig. 1)

Randhawa, M.S. 1959, P. 339, Fig. 336

Vegetative cells $150-190 \mu \mathrm{m}$ long, $110-130 \mu \mathrm{m}$ broad, with plane end walls; chloroplasts $4-8$, conjugation scalariform, tubes formed by both gametangia; empty cells $140-200 \mu \mathrm{m}$ long, 110 $140 \mu \mathrm{m}$ broad; fruiting cells cylindrical, $180-240$ $\mu \mathrm{m}$ long, $135-140 \mu \mathrm{m}$ broad; zygospores ellipsoid, 150-170 $\mu \mathrm{m}$ long, 75-125 $\mu \mathrm{m}$ broad; median spore wall thick, minutely verrucose, with coarsely meshed reticulate ridges, brown.

Locality: Planktonic in Baghjhoda Jhill, 137 m, Khorsane, Morang district.

Collection number and date: EN 309, 17-112004.

Distribution: India.

Genus: Sirogonium Kützing 1843

Filaments simple, unbranched; vegetative cells cylindrical, 2-6 times as long as broad, with plane end walls and without external pectic layer; chloroplasts $2-9$, straight or slightly spiral, rarely more than $1 / 2$ a turn; otherwise vegetatively similar to Spirogyra.

Reproduction by zygospore; conjugation direct between gametangia, without conjugation tubes; progametangia divide into unequal gametangiasmall as male and large as female, rarely progametangia conjugate without division, flexing of filaments during conjugation; zygospores ellipsoid or ovoid; median spore wall thick, smooth or variously ornamented, yellow, brown or black.

Key to the species:

1. Median spore wall reticulate with scattered protuberances, zygospores $90-94 \mu \mathrm{m}$ broad, chloroplasts $6-9$, vegetative cells $76-80 \mu \mathrm{m}$ broad -- illinoiense (1)

1. Median spore wall smooth, zygospores $60 \mu \mathrm{m}$ broad, chloroplasts $3-6$, vegetative cells $45-48$ $\mu \mathrm{m}$ broad S. sticticum (2)

Sirogonium illinoiense (Transeau) G.M. Smith 1933 (Pl. 2, Fig. 4)

Tiffany, L.H. and M.E. Britton 1952, P. 164, Pl. 50, Figs. 529-530; Randhawa, M.S. 1959, P. 426, Fig. 511

Vegetative cells $230 \mu \mathrm{m}$ long, $76-80 \mu \mathrm{m}$ broad; chloroplasts $6-9$, nearly straight or upto 1 turn; conjugation direct; both gametangia more or less reflexed; fruiting cells inflated to $130 \mu \mathrm{m}$; zygospores ellipsoid, 155-160 $\mu \mathrm{m}$ long, 90-94 $\mu \mathrm{m}$ broad; median spore wall with scattered protuberances connected by a more or less prominent reticulum, yellow.

Locality: Planktonic in the stagnant water of Rawa river at Katahare Bensi, 720 m, Khotang district.

Collection number and date: EN 180, 19-052003.

Distribution: U.S.A.

Sirogonium sticticum (Engl. Bot.) Kützing 1843 (P1. 2, Fig. 5)

Prescott, G.W. 1951, P. 321, Pl. 76, Figs. 5-7; Tiffany, L.H. and M.E. Britton 1952, P. 162, Pl. 50, Fig. 531; Randhawa, M.S. 1959, P. 424, Fig. 508

Vegetative cells $150 \mu \mathrm{m}$ long, $45-48 \mu \mathrm{m}$ broad; chloroplasts $3-6$, nearly straight or $1 / 2$ a turn; conjugation direct; gametangia usually shortened, more or less reflexed; fruiting cells inflated to 75 $\mu \mathrm{m}$; zygospores ellipsoid, sometimes more or less ovoid, $89 \mu \mathrm{m}$ long, $60 \mu \mathrm{m}$ broad; median spore wall smooth, yellow.

Locality: Free-floating in the Pitchhra canal, $72 \mathrm{~m}$, Biratnagar, Morang district.

Collection number and date: EN 126, 26-032003.

Distribution: Africa, Australia, India (Banaras, Fyzabad), Italy (Pavia), South America, U.S.A. 

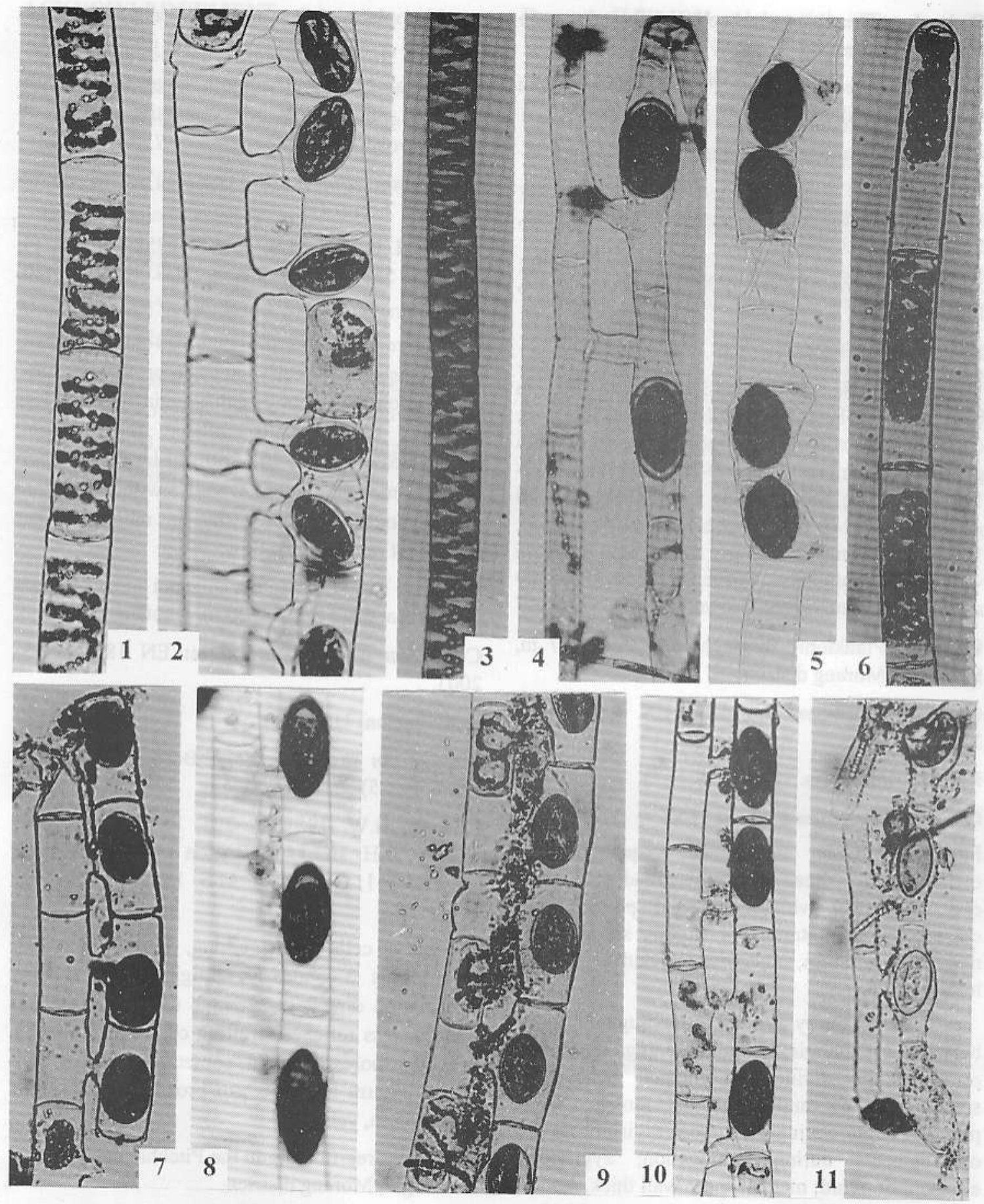

Plate 1. (Figs. 1-2. Spirogyra nugulosa Iwanoff, Figs. 3-4. S. esthonica (Skuja) Czurda, Fig, 5. S. daedaleoides Czurda, Figs. 6-7. S. rhizopus Jao, Fig. 8. S. communis (Hassal) Kützing, Fig. 9. S. decimina (Müller) Kützing, Fig. 10. S. juergensii Kützing, Fig. 11. S. pratensis Transeau). (All figures $\times 200$ magnification) 

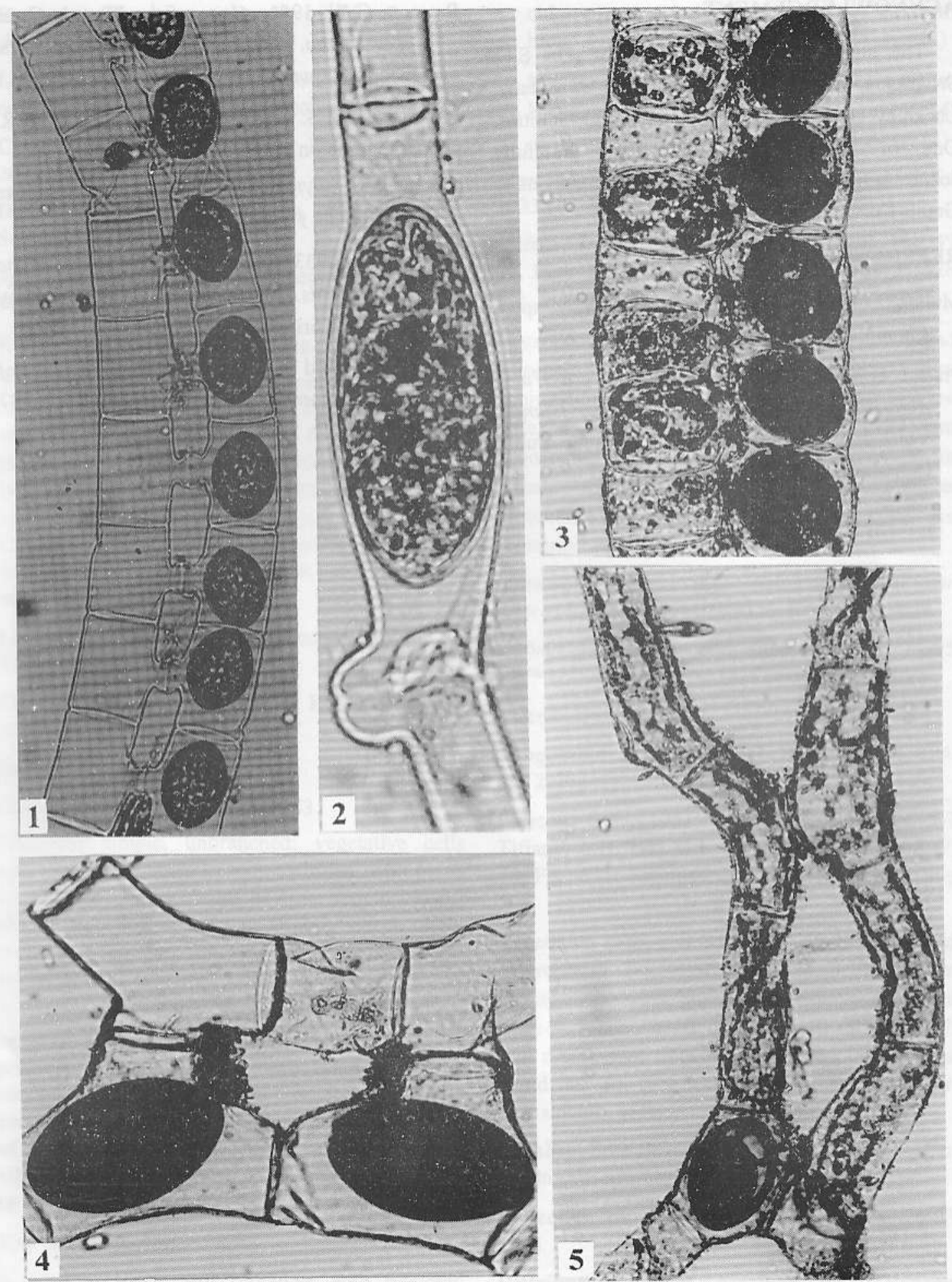

Plate 2. (Fig. 1. Spirogyra verrucosa (Rao) Krieger $\times 75$, Fig. 2. S. spreeiana Rabenhorst's $\times 750$, Fig. 3. S. paradoxa Rao $\times 200$, Fig. 4. Sirogonium illinoiense (Transeau) G.M. Smith $\times 200$, Fig. 5. S. sticticum (Engl. Bot.) Kützing $\times 200$ ). 


\section{ACKNOWLEDGEMENT}

The authors are thankful to Prof. Dr. S.C. Srivastava, retired Head, Department of Botany, Lucknow University, India for help and facilities. One of the authors (S.K. Rai) gratefully acknowledges financial assistance from University Grants Commission, Nepal.

\section{REFERENCES}

Müller, J. 1965. Spirogyra nepalensis nov. spec. Khumbu Himal. 1(4):299-300.

Prasad, B.N. and P.K. Misra 1992. Fresh Water Algal Flora of Andaman and Nicobar Islands, Vol. 2, B. Singh and M.P. Singh Publ., Dehradun, India. 284p.
Prescott, G.W. 1951. Algae of the Western Great Lakes Area. WM.C. Brown Publishers, Dubuque, Iowa. 977p.

Randhawa, M.S. 1959. Zygnemaceae. I.C.A.R. monograph on algae, New Delhi. 478p.

Rattan, R.S. 1967. Notes on some zygnemataceae from Punjab. Phykos. 6(1-2):95-99.

Smith, G.M. 1933. The Fresh Water Algae of the United States. McGraw-Hill Book Company Inc., New York. 716 pp.

Tiffany, L.H. and M.E. Britton 1952. The Algae of Illinois. Hafner Publishing Co., New York. 407 pp. 